\title{
Imagerie multispectralle dans la lutte anti-contrefaçon
}

\author{
Thomas Estruch, Bruno Pellegeay \\ Photonlines \\ th-estruch@photonlines.com
}

Face à la mondialisation et à la recrudescence de la contrefaçon, les sécurités mises au point pour protéger les produits se doivent d'être toujours plus performantes et difficiles à contrefaire. Les sciences forensiques en charge de détecter les faux des originaux doivent donc s'équiper à la pointe de la technologie de manière à lutter efficacement contre ce fléau mondial. Cet article a pour but de présenter les principales méthodes de spectro-imagerie pour la sécurité et l'identification de faux dans la lutte anti-contrefaçon.

$\mathrm{L}^{2}$ contrefaçon se définit comme l'activité de fabriquer un produit qui ressemble à un autre dans le but de le concurrencer ou de tromper le consommateur. La ressemblance objective entre les deux produits oblige un examen attentif, souvent effectué par des experts pour distinguer l'original de la copie. Si les premières traces de flibusterie recensées consistaient en la contrefaçon de fèves de cacao qui servaient de monnaie chez les Mayas, aujourd'hui la contrefaçon touche de nombreux domaines comme la cosmétique, la fabrication de produits pharmaceutiques et la falsification de documents officiels avec des conséquences parfois dramatiques pour la santé des consommateurs. La contrefaçon représente ainsi environ $5 \%$ du commerce mondial pour un coût de plus de 250 milliards de dollars [1]. La lutte contre ce fléau est d'autant plus difficile que la mondialisation a considérablement fait augmenter le volume des échanges et le progrès technologique a rendu les copies de plus en plus difficiles à repérer. Les sciences «forensiques » doivent donc être équipées de la pointe de la technologie en matière de spectro-imagerie pour garder un (ou plusieurs) coups d'avance sur les pirates modernes.

\section{Quels sont les types \\ de sécurités existantes?}

Si la détection des contrefaçons passe en partie par l'éducation et la vigilance du consommateur (variété des formats et couleurs dans les billets de banque par exemple), il existe également des protections mises en place pour lutter contre ce phénomène qui se décomposent en trois niveaux [2] (Tableau 1).

\section{Dispositif à variations optiques (OVD) [3]}

Les Exelgrammes ont été reconnus en 1995 comme l'une des protections les plus sûres pour lutter contre la contrefaçon. Mise au point au début des années 70 , cette technologie est basée sur la microstructuration d'une image par de multiples réseaux diffractifs curvilignes et interconnectés pour obtenir une coloration qui dépend de l'angle et de la quantité de lumière incidente. La volonté de transformer en OVD n'importe quel objet graphique a donné lieu à l'invention du Pixelgramme. Évolution de l'Exelgramme, chaque pixel de l'image va alors posséder son propre réseau de diffraction dont la période va déterminer la couleur du pixel et la courbure des lignes, l'intensité du pixel. Une image étant généralement de l'ordre de $1000 \times 1000$ pixels et chaque pixel étant composé d'un réseau curviligne échantillonné par un maillage de 1000 x 1000 points dont les positions sont précises, cela représente un motif complexe composé de réseaux diffractifs plus ou moins courbés de plus d'un trilliard de points !!! On comprend alors toute la difficulté de contrefaire un tel motif. Chaque point de ce maillage faisant environ $30 \mathrm{~nm}$ de diamètre, la seule technique envisageable

Tableau 1. Type de sécurité par niveau.

Niveau 1

- Dispositif à variations optiques * (OVD)

- Tatouage numérique (watermark)

- Encre à réponse optique variable (OVI)

- Motifs en transvision

- Signature et tampons
Niveau 2

- Encres UV mono/vari chromatique (350 nm), blanches polyspectrales (250 nm-350 nm), magnétisable, infrarouge

- Images latentes

- Micro impression *
Niveau 3

- Microparticule d'identification (microtaggant)

- Ruban/fil de sécurité UV

- Encres anti-stokes

- Informations personnelles invisibles * (IPI) 
pour la fabrication de ces motifs est la lithographie électronique.

\section{Les effets d'impression [4]}

Qu'il s'agisse de microimpressions ou d'images latentes (variation de couleur ou la trame du motif), l'objectif est de rendre un document impossible à numériser à l'aide d'un scanner. Les motifs très résolus sont alors difficiles à reproduire car ils nécessitent l'utilisation d'imprimantes très bien réglées et de scanners de très haute

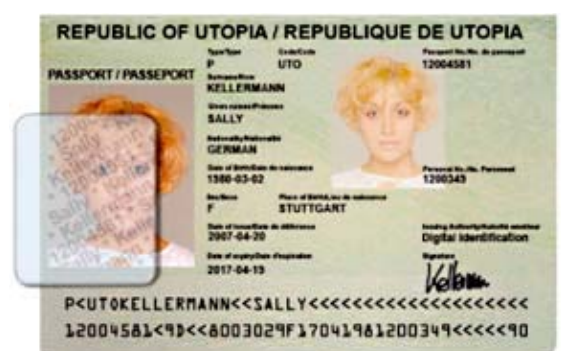

Figure 1. Exemple d'informations personnelles invisibles (IPI) encapsulées dans une carte d'identité. résolution. On peut également associer ces techniques à I'utilisation d'encres UV blanches polyspectrales qui vont se révéler blanches légèrement transparentes et brillantes à l'œil nu mais qui vont, sous l'effet d'une illumination UV entre $250 \mathrm{~nm}$ et $350 \mathrm{~nm}$, faire apparaître des motifs colorés rouge fluorescent ou vertjaune. On trouve également des encres infrarouges opaques ou transparentes dans le visible très onéreuses et donc encore peu utilisées par les faussaires.

\section{Informations personnelles invisibles (IPI)}

Un peu à l'image du watermark qui consiste à introduire des informations liées à l'acheteur en les encodant dans le produit sous forme de filigrane, les IPI correspondent aux informations personnelles invisibles à l'œil nu (date de naissance, nom, prénom, numéro de passeport etc.) encodées à l'aide d'un algorithme dans le document (figure 1). Pour pouvoir authentifier le document il faut alors utiliser un décodeur qui va mettre en évidence sous illumination UV les données avant de les décrypter à l'aide d'un algorithme particulier dont la clé de codage n'est connue que des services forensiques et est personnalisable pour chaque produit.

\section{L'analyse dans la contrefaçon}

Si ces différentes sécurités permettent de protéger les produits contre la contrefaçon, elles n'empêchent toutefois pas les essais de flibusterie. Ainsi le marché mondial est inondé de copies plus ou moins bien réalisées qu'il faut être capable d'analyser et d'identifier en tant que faux.

\section{Reconnaissance de formes et caractères}

Il existe toute une panoplie d'outils de mesure logiciels qui permettent de mesurer la conformité d'un caractère par rapport à une référence. Pour chaque police d'écriture, la taille des caractères, leur aire, l'épaisseur du trait, leur position sur la ligne ainsi que leur inclinaison sont
HORIBA

Scientific

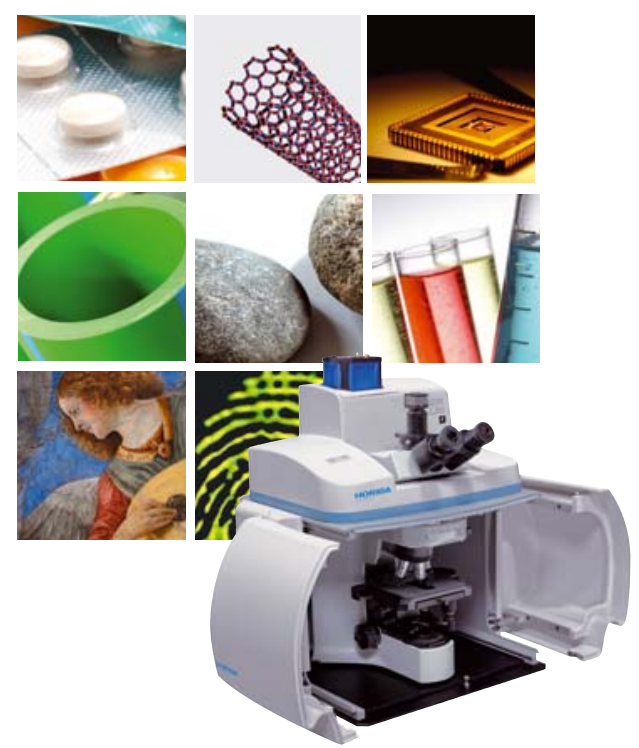

Imagerie et Analyse Physico-Chimique sans Préparation, en «1 click»

$\checkmark$ Analyse non-destructive et sans contact

$\checkmark$ Sans préparation d'échantillon

$\checkmark$ Temps d'analyse rapide et haut rendement

$\checkmark$ Analyse spectroscopique performante : détails, résolution et domaine d'analyse

$\checkmark$ Acquisition Raman «One Click»

$\checkmark$ Démarrage rapide, analyse possible 10 minutes après allumage de l'instrument

XploRA PLUS, L’imagerie Raman n'a jamais été aussi rapide ! 



Laser induced breakdown spectroscopy (LIBS) [7]

En focalisant un laser impulsionnel (femtoseconde à nanoseconde) sur un échantillon à analyser qu'il soit conducteur ou isolant, il est possible d'analyser sa structure chimique. Si la densité surfacique de puissance (éclairement de l'ordre de plusieurs centaines voire milliers de $\mathrm{MW} / \mathrm{cm}^{2}$ ) dépasse le seuil d'ablation $\mathrm{du}$ matériau, la matière n'a pas le temps d'évacuer la chaleur induite par conduction thermique (par le biais des phonons) et s'accumule alors dans une faible épaisseur de l'échantillon. Cet excédent d'énergie induit la fusion puis la vaporisation des atomes constituant l'échantillon qui vont être excités par collision et de manière radiative. En changeant la longueur d'onde du laser on ajuste la longueur de pénétration dans le matériau. Sous l'effet du refroidissement du plasma, les atomes et ions présents vont émettre un rayonnement caractéristique qui va être capté ensuite chargée électrostatiquement par une électrode à $7 \mathrm{kV}$. Dans les zones dites « douces » ou non imprimées, les charges vont alors se répartir uniformément alors que dans les zones contenant des impressions, les charges vont être perturbées ce qui va créer une image latente. Pour visualiser cette image latente, une encre en poudre (ou toner) est saupoudré sur le PET et va s'agglomérer dans les zones porteuses d'information. Cette impression est ensuite rendue permanente par collage d'une feuille plastique transparente sur le résultat du foulage. Cette technique permet de révéler des motifs sous-jacents lors du contact de plusieurs feuilles entre elles (jusqu'à 70 pages suivant le type de papier) mais également des variations de pression lors de l'impression du motif ce qui peut révéler la présence d'une contrefaçon.

\section{Spectroscopie Raman [6]}

La spectroscopie Raman consiste à étudier la composition moléculaire d'un échantillon de manière non destructive en se basant sur le principe qu'un substrat éclairé par une lumière monochromatique peut échanger de l'énergie avec les photons incidents (figure 3). On parle alors de diffusion inélastique. Les photons diffusés après réflexion sur le substrat sont alors

collectés par une lentille et dirigés vers un monochromateur qui va donner une information quantitative pour chaque longueur d'onde. Le spectre obtenu est composé de raies fines qui sont liées aux vibrations fondamentales des éléments chimiques qui constituent l'échantillon. On peut ainsi en effectuant une analyse Raman d'un médicament ou d'une encre par rapport à une référence, vérifier de manière qualitative et quantitative si l'échantillon est un original ou une contrefaçon.

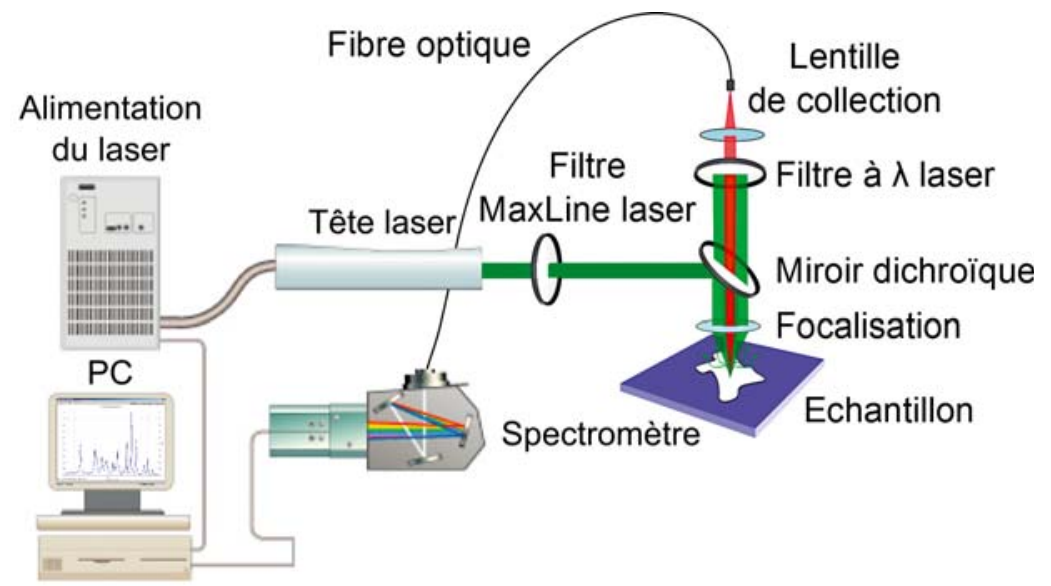

Figure 3. Schéma de principe de la spectroscopie Raman (méthode non destructive).

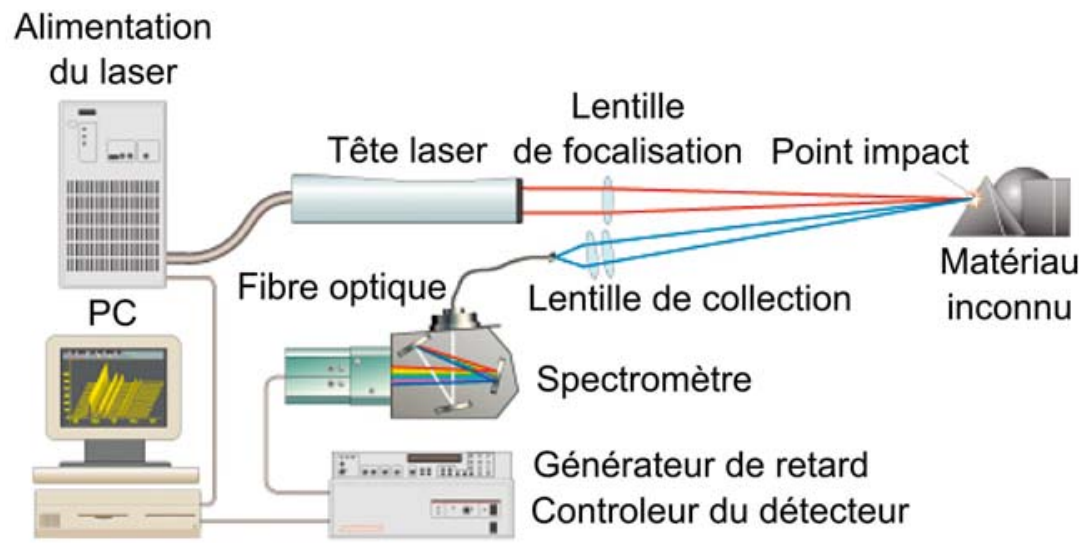

Figure 4. Fonctionnement du LiBS. 
par un spectromètre via un dispositif de collection optique. On obtient alors un spectre de raies entre $200 \mathrm{~nm}$ et $800 \mathrm{~nm}$ qui permet de déterminer qualitativement et quantitativement (par comparaison avec des échantillons étalons de concentrations connues) la composition élémentaire du plasma, donc de l'échantillon. Cette technique de mesure portable, bien que destructive pour l'échantillon, permet l'analyse quasi instantanée (quelques microsecondes) de quasiment la totalité des éléments du tableau périodique et est donc idéale pour la lutte anti-contrefaçon.

\section{La lutte anti-contrefaçon dans un futur proche?}

Une des difficultés de la lutte anti-contrefaçon provient du fait qu'il est difficile de former toutes les équipes sur le terrain à l'utilisation de matériel de laboratoire tels que des spectromètres ou des microscopes pour déterminer si un produit est une contrefaçon ou non. La nouvelle approche envisagée pour la lutte anti-contrefaçon consiste à imager en haute résolution le produit douteux sous différentes illuminations. Les images ainsi acquises sont ensuite envoyées à un centre d'expertise forensique via une connexion internet de manière à ce qu'un expert puisse les analyser, zoomer sans perte de résolution et rendre son jugement. Outre l'avantage de favoriser la collaboration entre les agents de terrain et les laboratoires d'analyse, cette nouvelle approche rend la lutte anti-contrefaçon plus réactive et temps réel en mettant en commun l'ensemble des bases de données internationales.

Tableau 1. Tableau récapitulatif des techniques de lutte anti-contrefaçon majoritairement utilisées en fonction du domaine d'application.

\begin{tabular}{|c|c|c|c|c|c|c|c|}
\hline & Médicaments & Documents & $\begin{array}{l}\text { Documents } \\
\text { identités }\end{array}$ & Bijoux & Parfums & Textiles & Aliments \\
\hline $\begin{array}{l}\text { Dispositif à } \\
\text { réponse optique } \\
\text { variable (OVD) }\end{array}$ & & $x$ & $x$ & & & & \\
\hline $\begin{array}{l}\text { Encres à réponse } \\
\text { optique } \\
\text { variable (OVI) }\end{array}$ & & $x$ & $X$ & & & $x$ & \\
\hline $\begin{array}{l}\text { Informations } \\
\text { personnelles } \\
\text { invisibles (IPI) }\end{array}$ & & & $X$ & & & & \\
\hline $\begin{array}{l}\text { Reconnaissance } \\
\text { de formes/ } \\
\text { caractères }\end{array}$ & $x$ & $x$ & $x$ & & & $x$ & $x$ \\
\hline Foulage & & $x$ & & & & & \\
\hline $\begin{array}{l}\text { Spectroscopie } \\
\text { Raman }\end{array}$ & $x$ & $x$ & & $x$ & $x$ & $x$ & $x$ \\
\hline LIBS & $x$ & $x$ & & $x$ & & & \\
\hline
\end{tabular}

\section{Références}

[1] http://www.diplomatie.gouv.fr/fr/politique-etrangere-de-la-france/defense-et-securite/criminaliteorganisee/contrefacon-16826/

[2] http://www.fosterfreeman.com/index.php/tech/3rd-level-security-features

[3] http://www.csiropedia.csiro.au/display/CSIROpedia/Optically+Variable+Devices

[4] http://digital.lgcforensics.com/services/questioned-documents/electrostatic-detection-apparatus. html [5] http://cerig.efpg.inpg.fr/memoire/2006/procedes-anti-contrefacon.htm\#marquagecontrefacon_arme-systèmes

[6] http://bictel.ulg.ac.be/ETD-db/collection/available/ULgetd-09102011-111908/unrestricted/ DOC4_Chapitre2.pdf

[7] http://www.libs-france.com/index.php/qu-est-ce-que-la-libs/le-principe

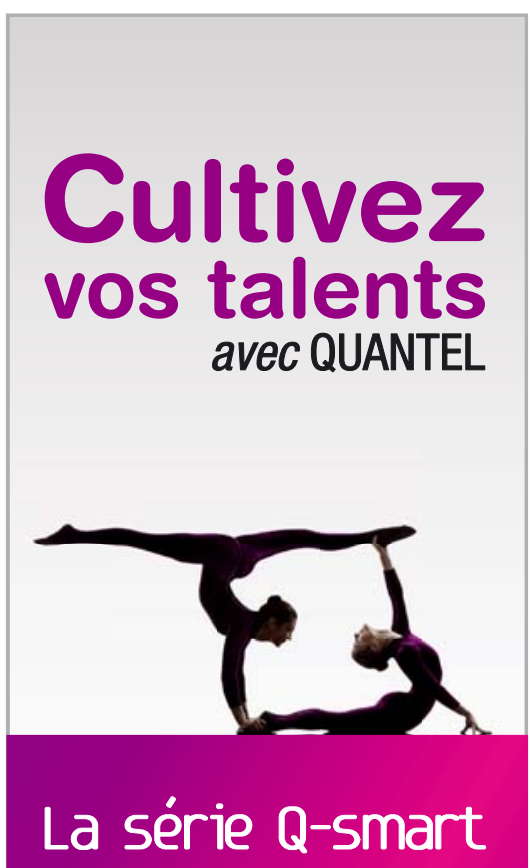

Les nouveaux lasers Nd:YAG pulsés par Quantel

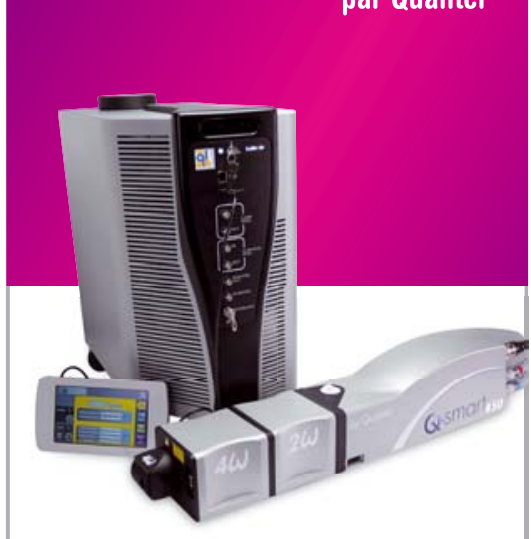

- Changement de longueur d'onde très simple

- Connexion rapide

- Légèreté et compacité

\section{(9l) Quantel}

quantel@quantel-laser.com www.quantel-laser.com 